\title{
STAPHYLOCOCCUS AUREUS- HOSPITAL ACQUIRED AND COMMUNITY ACQUIRED, AND ANTIMICROBIAL TESTING IN A TERTIARY CARE HOSPITAL
}

\author{
Padmavathy $M^{1}$, Priyanka $S^{2}$, Malini $J^{3}$
}

${ }^{1}$ Specialist cum Assistant Professor, Department of Microbiology, ESIC-Medical College-Postgraduate Institute of Medical Sciences and Research, Bangalore, Karnataka, India.

${ }^{2}$ Lady Medical Officer, Shivsamudra Hydrel Projects, Karnataka Power Corporation Limited, Shivanasamudram. Karnataka. 3Specialist cum Assistant Professor, Department of Microbiology, ESIC-Medical College-Postgraduate Institute of Medical Sciences and Research, Bangalore, Karnataka, India.

\section{BACKGROUND}

\section{ABSTRACT}

Staphylococcus aureus is a major pathogen causing a diversity of infections including bacteraemia, pneumonia, skin and soft tissue and osteoarticular infections. Infections due to MRSA are associated with increased morbidity and mortality in hospitalised patients. It also has the potential to cause sudden outbreaks in hospitals. Cost of treatment is another major problem found by patients in developing countries.

Aims and Objectives-

1. To differentiate MRSA into Hospital Acquired (HA) MRSA and Community Associated (CA) MRSA.

2. To know the antimicrobial susceptibility pattern of MRSA and Methicillin Sensitive Staphylococcus aureus (MSSA)

\section{MATERIALS AND METHODS}

This was a descriptive study. A total of 150 clinical isolates of Staphylococcus aureus from pus/ wound, blood, sputum, indwelling urinary catheter and urine during Nov 2011 to January 2013 were included. The S. aureus isolates were labelled as MRSA/ MSSA using cefoxitin ( $30 \mathrm{ug}$ ) discs and as hospital acquired and community acquired based on CDC criteria.

\section{RESULTS}

Out of 150 samples, 74 were MRSA and 76 were MSSA. Out of 74 MRSA samples, 51 were isolated from male and 23 were isolated from female. Out of 51 males, 41 were hospital acquired and 10 were community acquired. Out of 23 females, 21 were hospital acquired and 2 were community acquired.

\section{CONCLUSION}

Methicillin resistant S. aureus is a health risk in the community and to patients. HA-MRSA is an infection that can cause serious complications. This study emphasises the need for surveillance of patients who are admitted with HA-MRSA infection. This has important implication for MRSA epidemiology, infection control practices and empiric antimicrobial drug selection.

\section{KEY WORDS}

Staphylococcus, CA-MRSA, HA-MRSA.

HOW TO CITE THIS ARTICLE: Padmavathy M, Priyanka S, Malini J. Staphylococcus aureus- hospital acquired and community acquired, and antimicrobial testing in a tertiary care hospital. J. Evolution Med. Dent. Sci. 2018;7(40):4387-4391, DOI: $10.14260 /$ jemds/2018/978

\section{BACKGROUND}

Staphylococcus aureus is a major pathogen causing a diversity of infections ${ }^{1}$ including bacteraemia, pneumonia, skin and soft tissue and osteoarticular infections. In the past few decades, the prevalence of methicillin resistant $\mathrm{S}$. aureus among both nosocomial and community acquired infections has increased throughout the world.2

It is the most common cause of surgical wound infections and second only to coagulase-negative staphylococci as a cause of nosocomial blood stream infections. ${ }^{3}$ Staphylococci are among the hardest non-spore forming bacteria and they can survive many non-physiologic environmental conditions.

'Financial or Other Competing Interest': None.

Submission 07-03-2018, Peer Review 16-09-2018,

Acceptance 22-09-2018, Published 01-10-2018.

Corresponding Author:

PriyankaS,

Lady Medical Officer,

Shivsamudra Hydrel Projects,

Karnataka Power Corporation Limited,

Shivanasamudram, Bangalore, Karnataka, India.

E-mail: padmavathy1812@gmail.com

DOI: $10.14260 /$ jemds $/ 2018 / 978$

They can be cultured from dried clinical material after several months and are relatively heat resistant. ${ }^{4}$

Staphylococci are one of the major groups of bacteria inhabiting the skin, skin glands and mucous membranes. The skin and mucous membrane offer a very efficient mechanical barrier against local tissue invasion. If this barrier is breached by trauma or surgery, staphylococci will gain access to underlying tissue and create characteristic lesions such as abscess, impetigo, boils, folliculitis, carbuncles, cellulitis, furuncles, osteomyelitis etc.

Infections caused by $S$. aureus used to respond to $\beta$ lactam and related group of antibiotics. However, due to the development of multidrug resistance among S. aureus isolates, treatment of these infections has become problematic. $5^{5}$

Penicillin was successfully used to treat S. aureus infections till 1940. In 1942, penicillin resistant S. aureus infection appeared. Now 70 to $80 \%$ of S. aureus isolates are resistant to penicillin. Methicillin and other semisynthetic penicillin were successful in treating penicillin resistant S.aureus infections. ${ }^{3}$

Ever since its first isolation in 1961, MRSA has emerged as one of the commonest causes of Hospital Acquired 
Infection (HAI) and continues to remain an important factor contributing in failure of management. ${ }^{6}$

Infections due to MRSA are associated with increased morbidity and mortality in hospitalised patients. It also has the potential to cause sudden outbreaks in hospitals. Cost of treatment is another major problem found by patients in developing countries. ${ }^{6}$

Until recently, all strains of MRSA remained susceptible to vancomycin making this the drug of choice for treating infections caused by suspected or proven methicillin resistant staphylococci. However, after more than 30 years of Vancomycin use, resistance to it has emerged in clinical isolates. In May 1996, the World's first documented clinical infection due to Staphylococcus aureus with intermediate resistance to vancomycin was diagnosed in a patient in Japan. ${ }^{3}$

\section{Aims and Objectives of the Study}

1. To differentiate MRSA into Hospital Acquired (HA) MRSA and Community Associated (CA) MRSA.

2. To know the antimicrobial susceptibility pattern of Methicillin Resistant Staphylococcus aureus (MRSA) and Methicillin Sensitive Staphylococcus aureus (MSSA).

\section{MATERIALS AND METHODS}

This was a descriptive study conducted between November 2011 and January 2013 in the Department of Microbiology, ESIC MC, PGIMSR, Rajajinagar, Bangalore. Clinical sample like pus, blood, urine, sputum, body fluids, central venous catheter tips and indwelling urinary catheter was collected from the inpatients and outpatients of various departments of ESIMC and PGIMSR.

\section{Inclusion Criteria for CA-MRSA}

1. If it occurs in outpatient setting/ if a positive culture for MRSA is identified within $48 \mathrm{hrs}$. after admission to the hospital.

2. If there is no medical history in the past year of hospitalisation, admission to a nursing home, skilled nursing facility or hospice, dialysis or surgery.

3. If there are no permanent indwelling catheters or medical devices that pass through the skin into the body.

\section{Inclusion Criteria for HA-MRSA ${ }^{7}$}

1. If it occurs $>48 \mathrm{hrs}$. after admission to hospital.

2. If there is a history in the past year of hospitalisation, admission to the nursing home, skilled nursing facility or hospice, dialysis or surgery.

3. If there are any indwelling catheters or medical devices that pass through the skin into the body.

Staphylococcus aureus was identified according to the standard microbiological techniques (Koneman). Further MRSA and MSSA was differentiated by disc diffusion method using Cefoxitin (30 ug) disc. The diameter of zone of inhibition was measured with the help of scale and compared with CLSI zone size interpretative chart.8-9 Antimicrobial susceptibility testing of the isolated strains of Staphylococcus aureus to various antimicrobial discs was carried out according to Kirby-Bauer disc diffusion method. Antibiogram of MRSA and MSSA will be carried out using the panel of antibiotics as per CLSI (Clinical and Laboratory Standard Institute) 2010.

\section{RESULTS}

The present study was carried out at the Department of Microbiology, ESICMC, PGIMSR between November 2011 and January 2013. 150 clinical samples were collected from clinically suspected cases. Clinical diagnosis of the cases included in the present study were diabetic foot, cellulitis, osteomyelitis, non-healing ulcer, postoperative wound infection, gangrene, UTI, PUO, bronchitis, CSOM and burns. Various samples collected were pus/ wound swab, sputum, urine, tips of indwelling catheter, central venous line and tracheal tips.

Out of 150 subjects, 99 (66\%) patients were male and 51 (34\%) were female patients. The male-to-female ratio in the present study was 6.6: 3.4.

Majority of the patients were of age 21 - 30 years. The age and sex distribution are shown in Table 1 and 2.

\begin{tabular}{|c|c|}
\hline Age Group (Year) & No. of Cases \\
\hline$<11$ & 10 \\
\hline$>11-20$ & 13 \\
\hline$>21-30$ & 33 \\
\hline$>31-40$ & 25 \\
\hline$>41-50$ & 25 \\
\hline$>51-60$ & 27 \\
\hline$>61-70$ & 12 \\
\hline$>71$ & 5 \\
\hline \multicolumn{2}{|c|}{ Table 1. Age Distribution } \\
\hline \multicolumn{2}{|c|}{}
\end{tabular}

\begin{tabular}{|c|c|c|}
\hline Sex & Number & Percent \\
\hline Male & 99 & 66 \\
\hline Female & 51 & 34 \\
\hline Total & $\mathbf{1 5 0}$ & $\mathbf{1 0 0}$ \\
\hline \multicolumn{2}{|c|}{ Table 2. Sex Distribution } \\
\hline
\end{tabular}

Out of the total 150 samples 136 samples were pus, 6 miscellaneous, 5 sputum samples and 3 urine samples as shown in Table 3.

\begin{tabular}{|c|c|c|}
\hline Sample & Number & Percent (\%) \\
\hline Pus & 136 & 90.6 \\
\hline Miscellaneous & 6 & 4.0 \\
\hline Sputum & 5 & 3.3 \\
\hline Urine & 3 & 2.0 \\
\hline Total & $\mathbf{1 5 0}$ & $\mathbf{1 0 0}$ \\
\hline \multicolumn{3}{|c|}{ Table 3 } \\
\hline
\end{tabular}

The clinical conditions of 150 cases are shown in Table 4.

\begin{tabular}{|c|c|c|}
\hline Diagnosis & Number & Percent (\%) \\
\hline Abscess & 44 & 29.3 \\
\hline Injury/Wound & 33 & 22.0 \\
\hline Post-Op Wound & 16 & 10.6 \\
\hline Cellulitis & 12 & 8 \\
\hline Miscellaneous & 12 & 8 \\
\hline Osteomyelitis & 8 & 5.3 \\
\hline Ulcer & 6 & 4 \\
\hline Respiratory infection & 6 & 4 \\
\hline Diabetic foot & 5 & 3.3 \\
\hline CSOM & 5 & 3.3 \\
\hline UTI Table 4. Distribution of Cases \\
\hline \multicolumn{2}{|c|}{} \\
\hline
\end{tabular}

Out of 150 total samples, 74 were MRSA and 76 were MSSA. 


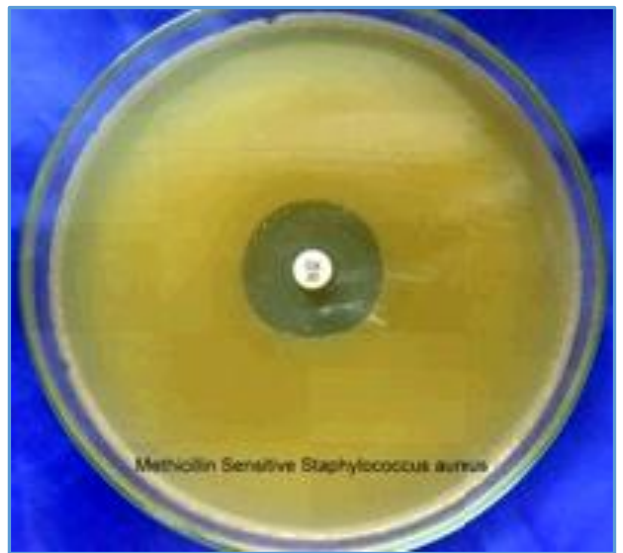

Figure $6 a$

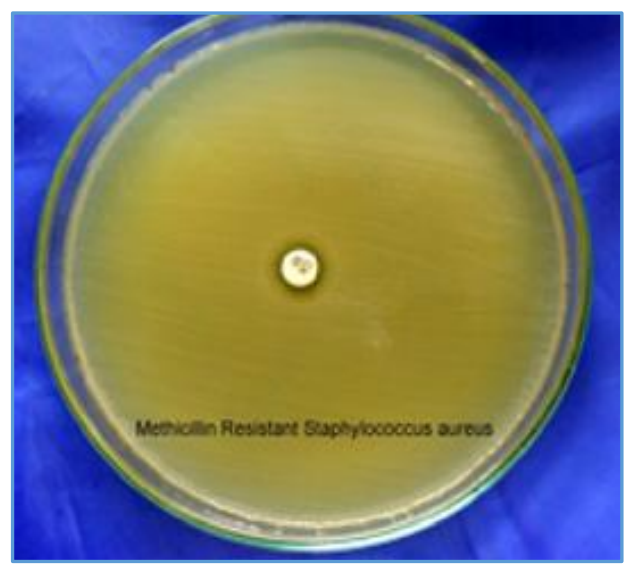

Figure $6 b$

Methicillin sensitive Staphylococcus aureus - Methicillin resistant Staphylococcus aureus out of 74 MRSA samples-

- 51 were isolated from male. Out of 51, 41 were hospital acquired and 10 were community acquired. More number of HA cases were seen in 21 - 30 years' age group and more number of CA seen in 31 - 40 and 51 - 60 years' age group.

- 23 were isolated from female. Out of 23,21 were hospital acquired and 2 were community acquired. More number of HA cases were seen in 51 - 60 years' age group and CA seen only in 21 - 30 years' age group.

\begin{tabular}{|c|c|c|}
\hline Organism & Number & Percentage \\
\hline MRSA & 74 & 49.3 \\
\hline MSSA & 76 & 50.6 \\
\hline Total & $\mathbf{1 5 0}$ & $\mathbf{1 0 0}$ \\
\hline \multicolumn{2}{|c|}{ Table 5. Differentiation of Total Samples based on } \\
Cefoxitin Resistance/Sensitivity
\end{tabular}

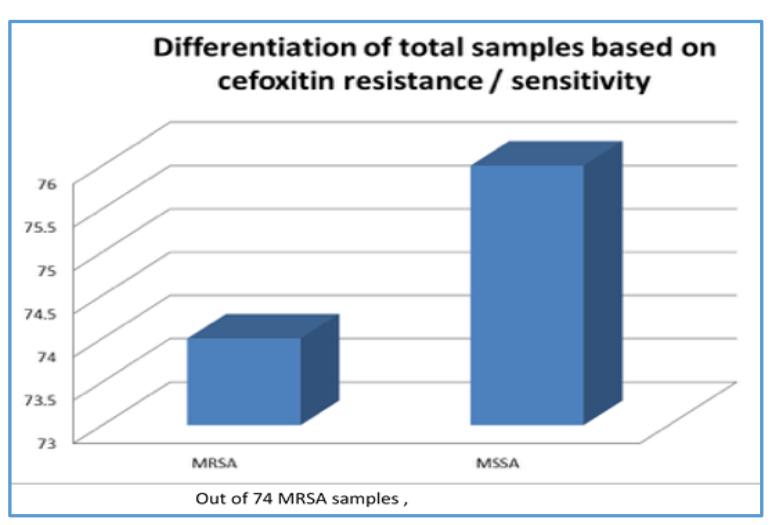

Graph 5. Differentiation of Total Samples based on Cefoxitin Resistance/Sensitivity
- $\quad 51$ were isolated from male. Out of 51, 41 were hospital acquired and 10 were community acquired. More number of HA cases were seen in 21 - 30 years' age group and more number of CA seen in 31 - 40 and 51 - 60 years' age group.

- 23 were isolated from female. Out of 23, 21 were hospital acquired and 2 were community acquired. More number of HA cases were seen in 51 - 60 years' age group and CA seen only in 21 - 30 years' age group.

\begin{tabular}{|c|c|c|c|c|}
\hline & \multicolumn{2}{|c|}{ Male } & \multicolumn{2}{c|}{ Female } \\
\hline Age & HA & CA & HA & CA \\
\hline$<10$ & 2 & 0 & 2 & 0 \\
\hline $11-20$ & 3 & 1 & 1 & 0 \\
\hline $21-30$ & 10 & 0 & 5 & 2 \\
\hline $31-40$ & 7 & 4 & 2 & 0 \\
\hline $41-50$ & 8 & 1 & 2 & 0 \\
\hline $51-60$ & 4 & 4 & 6 & 0 \\
\hline $61-70$ & 6 & 0 & 2 & 0 \\
\hline $71+$ & 41 & 10 & 21 & 2 \\
\hline
\end{tabular}

Table 6. Age Wise and Sex Wise Distribution of HA and CA MRSA

Out of 76 MSSA Samples,

- 48 were isolated from male. Out of 48,26 were hospital acquired and 22 were community acquired. More number of HA cases were seen in 21 - 30 years' age group and more number of CA seen in 41 - 50 years' age group.

- $\quad 28$ were isolated from female. Out of 28, 23 were hospital acquired and 5 were community acquired. More number of HA cases were seen in 31 - 40 years' age group.

\begin{tabular}{|c|c|c|c|c|}
\hline & \multicolumn{2}{|c|}{ Male } & \multicolumn{2}{c|}{ Female } \\
\hline Age & HA & CA & HA & CA \\
\hline$<10$ & 0 & 0 & 5 & 1 \\
\hline $11-20$ & 3 & 4 & 0 & 1 \\
\hline $21-30$ & 7 & 5 & 4 & 0 \\
\hline $31-40$ & 6 & 0 & 6 & 0 \\
\hline $41-50$ & 4 & 8 & 2 & 0 \\
\hline $51-60$ & 4 & 4 & 4 & 1 \\
\hline $61-70$ & 2 & 0 & 1 & 1 \\
\hline $71+$ & 0 & 1 & 1 & 1 \\
\hline Total & 26 & 22 & 23 & 5 \\
\hline Table 7. Age Wise and Sex Wise Distribution of HA and CA \\
MSSA \\
\hline
\end{tabular}

\section{DISCUSSION}

Staphylococcus aureus is a major pathogen responsible for nosocomial and community acquired infection and MRSA has emerged as a major nosocomial pathogen and an increasingly frequent cause of community acquired infections that cause significant morbidity and mortality. Biofilm production is an important factor which helps the organism to adhere onto surfaces, which facilitates later in invasion and causing infection. This study shows a significant difference between MRSA and MSSA in the production of biofilm $(p<0.001)$.

The present study shows Methicillin resistant Staphylococcus aureus and Methicillin sensitive Staphylococcus aureus and their antibiogram in clinical isolates was carried out at the Department of Microbiology, ESIC-MC and PGIMSR between November 2011 and January 2013.

For this purpose, 150 clinical samples were collected. Out of which, majority of strains were isolated from pus samples i.e. $136(90 \%)$ followed by $6(4 \%)$ miscellaneous, 5 (3.33) 
sputum and $3(2 \%)$ were from urine samples. Kumari et al ${ }^{10}$ reported $64 \%$ Staphylococcus from pus samples followed by $20 \%$ blood, $4.8 \%$ device associated and $3.2 \%$ from urine.

Male-to-female ratio was 6.6: 3.6. The increased rate of infection among males could be due to their outdoor occupation, more prone for injuries (fracture), smoking (gangrene) and due to exposure to contaminated environment (cellulitis). Occupationally males are more exposed as agriculturists and industrial employees. A similar observation has been made by Siddique et $\mathrm{al}^{11}$ who has reported a male-to-female ratio of 2.6: 1 . In the present study, out of 150 cases most of the cases were seen in young age, majority being between 21 - 30 years. This may be because of more prone for injuries (fracture) and due to exposure to contaminated environment (Cellulitis).

Methicillin resistance was detected by phenotypic method using cefoxitin $(30 \mu \mathrm{g})$ disc diffusion technique. Recent studies indicate that disc diffusion testing using cefoxitin disc is far superior to most of the currently recommended phenotypic methods like oxacillin disc diffusion and oxacillin screen agar testing and is now an accepted method for the detection of MRSA (CLSI).

In the present study, out of 150 samples cefoxitin disk sensitivity revealed that $74(49.33 \%)$ isolates were methicillin resistant Staphylococcus aureus (MRSA), 76 (50.66\%) were methicillin sensitive Staphylococcus aureus (MSSA) and predominant isolates were MSSA (50.66). Angel MR et al ${ }^{12}$ reported $11.11 \%$ MRSA, 24.32\% MSSA indicating predominance of MSSA.

There is a significant increase in the methicillin resistant isolates in our hospital compared with the study by Ajantha GS et al ${ }^{13}$ reported $33.64 \%$ MRSA and $66.35 \%$ MSSA. Krishna BVS et al ${ }^{14}$ reported $18.1 \%$ MRSA and $81.89 \%$ MSSA.

In the present study, majority $62(83.78 \%)$ MRSA were isolated from IPD patients and $12(16.21 \%)$ from OPD indicating higher percentage of infection by MRSA among hospitalised patients.

Similarly, Patrick M et al ${ }^{15}$ reported $76 \%$ MRSA from IPD and only $21 \%$ MRSA from OPD. Out of 76 MSSA isolates, $49(64.47 \%)$ were from hospital acquired and 27 (35.5\%) were community acquired indicating higher percentage of infection by MSSA among hospitalised patients.

Studies have shown that although there is no significant difference between rates of disseminated infection by MRSA and MSSA in hospitals, the mortality rate is significantly higher in infections caused by MRSA due to the added interference of antimicrobial resistance (Melzer et al, 2003). ${ }^{16}$

Out of 74 MRSA 62 (83.78\%) MRSA were hospital acquired, 12 (16.21\%) from community acquired. And Out of 76 MSSA isolates, $49(64.47 \%)$ were from hospital acquired and 27 (35.5\%) were from community acquired.

\section{CONCLUSION}

Methicillin resistant $\mathrm{S}$. aureus is a health risk in the community and to patients. HA-MRSA is an infection that can cause serious complications. This study emphasises the need for surveillance of patients who are admitted with HA-MRSA infection. This has important implication for MRSA epidemiology, infection control practices and empiric antimicrobial drug selection.

\section{REFERENCES}

[1] Denis 0, Nonhoff C, Byl B, et al. Emergence of vancomycin-intermediate staphylococcus aureus in a Belgian hospital: microbiological and clinical features. J Antimicrob Chemotherapy 2002;50(3):38391.

[2] NNIS System. National Nosocomial Infections Surveillance (NNIS) System Report, data summary from January 1990 - May 1999, Issued June 1999. A report from the NNIS system. American Journal of Infection Control 1999;27(6):520-32.

[3] Smith TL, Pearson ML, Wilcox KR, et al. Emergence of vancomycin resistance in staphylococcus aureus. Glycopeptide-Intermediate Staphylococcus aureus Working Group. New England Journal of Medicine 1999;340(7):493-501.

[4] Waldvogel FA. Staphylococcus aureus. In: Mandell GL, Bennett JE, Dolin R, eds. Principles and Practice of infectious diseases. Vol. 2. $5^{\text {th }}$ edn. Philadelphia: Churchill Livingstone 2000: p. 2069-92.

[5] Vidhani S, Mehndiratta PL, Mathur MD. Study of methicillin resistant staphylococcus aureus isolates from high risk patients. Ind J Med Microbiol 2001;19(2):13-6.

[6] Chaudary U, Anupama. Prevalence of methicillin resistance in staphylococcus aureus. Indian J Med Microbiol 1999;17(3):154-5.

[7] Centres for disease control and Prevention 2005, Feb 3 , community associated methicillin resistant staphylococcus aureus and hospital associated methicillin resistant staphylococcus aureus information for clinician retrieved Dec 20, 2007, sitedoct 112011 http://www.cdc.gov/ncidod/dhqp/av_MRSA_ca.clinici an.html.

[8] Koneman EW, Allen S, Janda W, et al. The gram positive cocci. Part I. Staphylococci and related organisms. Color Atlas and Text Book of Diagnostic Microbiology. $6^{\text {th }}$ edn. New York: Lippincott 2006: p. 624-79.

[9] National Committee for Clinical Laboratory Standards Methods for dilution antimicrobial susceptibility tests for bacteria that grow aerobically. NCCLS approved standards M2-A7. 2002.

[10] Kumari N, Mohapatra TM, Singh YI. Prevalence of Methicillin Resistant Staphylococcus Aureus (MRSA) in a tertiary care hospital in Eastern Nepal. J Nepal Med Assoc 2008;47(170):53-6.

[11] Siddiqui GM, Karamat KA, Hanan A. Prevalence of methicillin-resistant staphylococcus aureus: a study at PAF Hospital Sargodha. Pak J Pathology 1999;10:26-8.

[12] Angel MR, Balaji V, Prakash J, et al. Prevalence of inducible clindamycin resistance in gram positive organisms in a tertiary care centre. Indian J Med Microbiol 2008;26(3):262-4.

[13] Ajantha GS, Kulkarni RD, Jeevan S, et al. Phenotypic detection of inducible clindamycin resistance among staphylococcus aureus isolates by using the lower limit of recommended inter-disk distance. Indian J Pathol Microbiol 2008;51(3):376-8. 


\section{Jemds.com}

[14] Krishna BVS, Asha BP, Chandrasekhar MR. Communityacquired methicillin-resistance staphylococcus aureus infections in a South Indian city. Southeast Asian J Trop Med Public Health 2004;35(2):371-4.

[15] Schlievert PM, Strandberg KL, Lin YC, et al. Secreted virulence factor comparison between methicillinresistant and methicillin-sensitive staphylococcus aureus and its relevance to atopic dermatitis. J Allergy Clin Immunol 2010;125(1):39-49.

\section{Original Research Article}

[16] Melzer M, Eykyn SJ, Gransden WR, et al. Is methicillinresistant Staphylococcus aureus more virulent than methicillin - susceptible S. aureus? A comparative cohort study of British patients with nosocomial infection and bacteremia. Clin Infect Dis 2003;37(11):1453-60. 